\title{
Prevalence and associated factors of poor sleep quality among Chinese older adults living in a rural area: a population-based study
}

\author{
Peng Wang ${ }^{1,2} \cdot$ Lin Song $^{1} \cdot$ Kaili Wang $^{3} \cdot$ Xiaolei Han $^{1} \cdot$ Lin Cong $^{1} \cdot$ Yongxiang Wang $^{1} \cdot$ Lei Zhang $^{2} \cdot$ Zhongrui Yan $^{2}$. \\ Shi Tang ${ }^{1} \cdot$ Yifeng $\mathrm{Du}^{1}$
}

Received: 19 September 2018 / Accepted: 8 March 2019 / Published online: 27 March 2019

(c) The Author(s) 2019

\begin{abstract}
Objective To investigate the prevalence and associated factors of poor sleep quality among community-dwelling elderly population in a rural area of Northern China.

Methods We conducted a cross-sectional survey in August-December 2014 and recruited 2195 participants who were aged 65 years or older and living in Yanlou Town of Yanggu County in western Shandong Province, China. Data on demographics, health-related behaviors, and clinical conditions were collected through structured interviews. The Pittsburgh Sleep Quality Index (PSQI) was used to assess the sleep quality and patterns. Poor sleep quality was defined as a PSQI score $>7$. We employed multiple logistic models to relate poor sleep quality to various factors.

Results The overall prevalence rates of poor sleep quality were $33.8 \%$ in the total sample, $39.2 \%$ in women and $26.3 \%$ in men $(P<0.01)$. The most common abnormal sleep domains were prolonged sleep latency $(39.7 \%)$, decreased sleep duration (31.0\%), and reduced habitual sleep efficiency (28.8\%). Multiple logistic regression analyses revealed that poor sleep quality was significantly associated with female sex $(\mathrm{OR}=1.76,95 \% \mathrm{CI} 1.46-2.12)$ and clinical comorbidities such as hypertension $(\mathrm{OR}=1.28,95 \% \mathrm{CI} 1.06-1.54)$, coronary heart disease $(\mathrm{OR}=1.60,95 \% \mathrm{CI} 1.27-2.00)$, and chronic obstructive pulmonary disease (OR $=1.82,95 \%$ CI 1.34-2.49).

Conclusions The sleep disorders were highly prevalent among the elderly in rural China. Modifiable risk factors such as cardiometabolic risk factors and disorders were associated with poor sleep quality, which might be potential targets for interventions to improve sleep quality in elderly population.
\end{abstract}

Keywords Poor sleep quality · Pittsburgh Sleep Quality Index (PSQI) · Elderly population

\section{Introduction}

Peng Wang and Lin Song contributed equally to this work.

Shi Tang

Tangshi1989@163.com

$\triangle$ Yifeng Du

du-yifeng@hotmail.com

1 Department of Neurology, Shandong Provincial Hospital affiliated to Shandong University, No. 324 Jingwuweiqi Road, Jinan 250021, Shandong, People's Republic of China

2 Department of Neurology, Jining No. 1 People's Hospital, Jining, Shandong, People's Republic of China

3 Department of Traditional Chinese Medicine, Jining No. 1 People's Hospital, Jining, Shandong, People's Republic of China
In 2017, the number of people aged 60 years and above had reached nearly 241 million in China, accounting for $17.3 \%$ of the nation's total population [1]. Large-scale populationbased epidemiologic studies have demonstrated that sleep complaints are common in the elderly. For example, the Survey of Health Ageing and Retirement in Europe (SHARE) of people aged 50 years and older from 16 European countries showed that the prevalence of sleep problems varied from $\sim 17 \%$ in Denmark and Italy to $\sim 31 \%$ in Poland [2]. Furthermore, the US National Health Aging Trends Study (NHATS) found that $28 \%$ of the study participants (age $\geq 65$ years) experienced poor sleep quality [3]. The French Three-City Study of 9294 community-dwelling persons aged 65 years or older found that $30.9 \%$ of older adults suffered from 
insomnia complaints [4]. In addition, the prevalence of sleep disorders among residents aged $\geq 60$ years in northern and southern Chinese urban areas was $37.8 \%$ and $41.5 \%$, respectively $[5,6]$. Chronic sleep disorders were associated with poor health, and older people with sleep disorders are at marked risk of falls [7], disability [8], hospitalization [9], and mortality [10]. However, the prevalence data of sleep disorders among the elderly in rural areas of northern China are still lacking.

Evidence is emerging that the socio-demographic and lifestyle factors may play a role in sleep disorders among older adults. For instance, female gender, low education, divorce and widowhood, living alone, inadequate fruit intake, drinking tea, alcohol consumption, caffeine, use of certain medications, poor mental health, and physical inactivity have been reported to be associated with sleep disturbance [5, 6, 11-20]. In addition, poor sleep quality or sleep disorders in old people are associated with highly prevalent chronic health conditions, such as hypertension [21], type 2 diabetes [22], cardiovascular disease [23], stroke [24], depression [25], and cognitive impairment [26]. Most of these previous studies have been conducted in western societies. However, the socio-demographic, lifestyle, and medical factors of sleep disorders may differ across ethnic populations. Identifying potentially modifiable factors that are related to poor sleep quality or sleep disorders may help develop effective interventions to improve sleep quality and, thus reduce the risk of chronic diseases in older adults associated with sleep disorders [27].

Therefore, in this large-scale population-based study, we sought to investigate the prevalence of poor sleep quality and abnormal sleep patterns, and to further analyze a range of various factors related to poor sleep quality among older adults who were living in a rural area in western Shandong province, China.

\section{Methods}

\section{Study population}

Participants in this cross-sectional survey were from the Shandong Yanggu Study of aging and health that targeted elderly residents (age $\geq 65$ years) who were living in the rural area of Yanlou Town of Yanggu County in western Shandong province, China. From August through December 2014, 2986 (90.7\%) of the 3298 eligible participants were recruited. Of these persons, 791 were excluded due to missing data on sleep and other variables, leaving 2195 persons for the current analysis.

\section{Data collection}

Data were collected through face-to-face interviews by trained staff following a structured questionnaire. We collected data on socio-demographics (e.g., age, sex, education, marital status, and occupation), health-related behavioral factors (e.g., smoking and alcohol drinking), and health history [e.g., a physician's diagnosis of hypertension, diabetes, hyperlipidemia, coronary heart disease (CHD), chronic obstructive pulmonary disease (COPD), and stroke]. Education was divided into illiteracy, primary school ( $1-5$ years), and middle school and above ( $\geq 6$ years). Occupation was dichotomized into farmers vs. non-farmers. We categorized smoking status and alcohol consumption as current vs. noncurrent (never or former). Marital status was divided into married, unmarried, divorced, and widowed. History of each of those disorders was dichotomized as yes vs. no according to self-reported information.

\section{Assessments of sleep quality}

Sleep quality was measured with the Pittsburgh Sleep Quality Index (PSQI), which is a validated self-rated questionnaire that assesses sleep quality and sleep disturbance over a 1-month period [28]. PSQI includes 19 items that assess seven domains: subjective sleep quality, sleep latency, sleep duration, habitual sleep efficiency, sleep disturbances, use of sleep medication, and daytime dysfunction over the last month. Score for each domain ranges from 0 to 3 (no difficulty to severe difficulty). Scores for all seven domains are summed up to yield a global PSQI score, ranging from 0 to 21. Higher scores indicate worse sleep quality. We defined poor sleep quality as the overall PSQI score $>7$ [29]. The abnormal sleep in specific domain was defined as the score for that domain $\geq 2$. The Chinese version of the PSQI had good overall reliability $(r=0.82-0.83)$ and test-retest reliability $(r=0.77-0.85)[30]$.

\section{Statistical analysis}

We presented the sociodemographic characteristics, behavioral factors, and clinical conditions of participants by sleep disorders, and tested the statistical differences using the Chi square test for categorical variables and the $t$ test for normal distributed continuous variables. We employed logistic regression models to estimate the odds ratios and $95 \%$ confidence intervals of sleep disturbance associated with different factors, while adjusting for different potential confounding factors. We reported results from three models: model 1 was controlled for basic demographic factors (age, sex, and education); model 2 was additionally controlled for marital status, occupation, 
health-related behavioral factors (smoking and alcohol consumption), and clinical conditions (e.g., history of hypertension, hyperlipidaemia, diabetes, CHD, COPD, and stroke); and model 3 represented the best-fit model using the stepwise forward approaches. We considered two-tailed $p$ level $\leq 0.05$ to be statistically significant. IBM SPSS Statistics for Windows (version 24.0) (Armonk, NY, USA: IBM Corp) was used for all statistical analyses.

\section{Results}

\section{Characteristics of study participants}

Table 1 shows characteristics of the study participants by sleep quality (global PSQI score $>7$ vs. $\leq 7$ ). Of the 2195 participants, the mean age was 71.67 years (SD, 5.89 ) and $57.9 \%$ were women. Overall, the prevalence of poor sleep quality was $33.8 \%$ in the total sample, $26.3 \%$ in men, and 39.2 in women (for sex difference, $p<0.01$ ). Poor sleep quality was significantly $(p<0.05)$ or marginally $(0.05<p<0.07)$ associated with all the examined factors in Table 1.

\section{Distribution of poor sleep quality and subdomains}

Table 2 shows the distribution of abnormal Pittsburgh subdomains by age groups and sex. The prevalence rates of abnormal specific sleep domains were $39.7 \%$ for sleep latency, $31.0 \%$ for sleep duration, $28.8 \%$ for habitual sleep efficiency, $26.8 \%$ for daytime dysfunction, $23.2 \%$ for sleep disturbance, $18.0 \%$ for subjective sleep quality, and $4.5 \%$ for sleep medicine use. There was no significant difference in prevalence of the abnormal Pittsburgh sleep subdomains among various age subgroups, except habitual sleep efficiency that the prevalence of abnormal habitual sleep efficiency increased with increasing age $(p<0.001)$. Moreover, the prevalence of abnormal PSQI components was higher in women than in men in all sleep subdomains (Table 2).

\section{Associated factors of poor sleep quality}

Table 3 shows the association of poor sleep quality (defined as a PSQI score >7) with different demographic, behavioral, and clinical factors. When controlling for demographic factors, poor sleep quality was significantly associated with female sex, current smoking, hypertension, hyperlipidemia, CHD, COPD, and stroke (Table 3, model 1). When entering all variables examined in Table 3 into the model simultaneously, the associations of current smoking, hyperlipidemia, and stroke with poor sleep quality were diluted and became statistically non-significant (Table 3 , model 2 ). We
Table 1 Characteristics of the study participants by sleep quality $(n=2195)$

\begin{tabular}{|c|c|c|c|c|}
\hline \multirow[t]{2}{*}{ Characteristics } & \multirow{2}{*}{$\begin{array}{l}\text { Total } \\
\text { Sample }\end{array}$} & \multicolumn{3}{|c|}{ Poor sleep quality (PSQI score $>7$ ) } \\
\hline & & No & Yes & $p$ value \\
\hline No. of subjects & 2195 & $1454(66.2)$ & $741(33.8)$ & \\
\hline Male & 924 & $681(73.7)$ & $243(26.3)$ & $<0.001$ \\
\hline Age (years), mean (SD) & $71.67(5.89)$ & $71.46(5.69)$ & $72.08(6.24)$ & $<0.020$ \\
\hline Marital status, married & 1580 & $1065(73.2)$ & $515(69.5)$ & 0.065 \\
\hline Occupation, farmers & 1985 & $1305(89.8)$ & $680(91.8)$ & 0.129 \\
\hline \multicolumn{5}{|l|}{ Education } \\
\hline Illiteracy & 1080 & $680(46.8)$ & $400(54.0)$ & $<0.001$ \\
\hline Primary school & 740 & $493(33.9)$ & $247(33.3)$ & \\
\hline Middle school or above & 375 & $281(19.3)$ & $94(12.7)$ & \\
\hline Current smoking & 793 & $563(38.7)$ & $230(31.0)$ & $<0.001$ \\
\hline Current alcohol drinking & 771 & $552(38.0)$ & $219(29.6)$ & $<0.001$ \\
\hline Hypertension & 886 & $544(37.4)$ & $342(46.2)$ & $<0.001$ \\
\hline Hyperlipidemia & 363 & $221(15.2)$ & $142(19.2)$ & $<0.018$ \\
\hline Diabetes & 188 & $113(7.8)$ & $75(10.1)$ & $<0.063$ \\
\hline CHD & 421 & $229(15.7)$ & $192(25.9)$ & $<0.001$ \\
\hline COPD & 188 & $98(6.7)$ & $90(12.1)$ & $<0.001$ \\
\hline Stroke & 232 & $141(9.7)$ & $91(12.3)$ & 0.063 \\
\hline
\end{tabular}

Data are $n(\%)$, unless otherwise specified

PSQI Pittsburgh Sleep Quality Index, CHD coronary heart disease, COPD chronic obstructive pulmonary disease 
Table 2 Distributions of abnormal Pittsburgh Sleep Quality Index subdomains by age and sex

\begin{tabular}{|c|c|c|c|c|c|c|c|c|}
\hline \multirow[t]{2}{*}{ Age or sex } & \multirow{2}{*}{$\begin{array}{l}\text { No. of } \\
\text { partici- } \\
\text { pants }\end{array}$} & \multicolumn{7}{|c|}{ Abnormal Pittsburgh Sleep Quality Index subdomains ${ }^{\mathrm{a}}, n(\%)$} \\
\hline & & $\begin{array}{l}\text { Subjec- } \\
\text { tive sleep } \\
\text { quality }\end{array}$ & Sleep latency & Sleep duration & Sleep efficiency & Sleep disturbance & $\begin{array}{l}\text { Use of sleep } \\
\text { medications }\end{array}$ & Daytime dysfunction \\
\hline Total sample & 2195 & $395(18.0)$ & $871(39.7)$ & $680(31.0)$ & $633(28.8)$ & $510(23.2)$ & $98(4.5)$ & $589(26.8)$ \\
\hline \multicolumn{9}{|c|}{ Age group, years } \\
\hline $65-69$ & 1005 & $172(17.1)$ & $390(38.8)$ & $322(32.0)$ & $274(27.3)$ & $240(23.9)$ & $44(4.4)$ & $276(27.5)$ \\
\hline $70-74$ & 581 & $106(18.2)$ & $226(38.9)$ & $166(28.6)$ & $148(25.5)$ & $131(22.5)$ & $31(5.3)$ & $151(26.0)$ \\
\hline $75-79$ & 332 & $60(18.1)$ & $128(38.6)$ & 105 (31.6) & $107(32.2)$ & $73(22.0)$ & $12(3.6)$ & $86(25.9)$ \\
\hline$\geq 80$ & 277 & $57(20.6)$ & $127(45.8)$ & 87 (31.4) & $104(37.3)$ & $66(23.8)$ & $11(4.0)$ & $76(27.4)$ \\
\hline$p$ value & & 0.614 & 0.168 & 0.533 & 0.001 & 0.865 & 0.621 & 0.894 \\
\hline \multicolumn{9}{|l|}{ Sex } \\
\hline Male & 924 & $123(13.3)$ & $280(30.3)$ & 249 (26.9) & $216(23.4)$ & 200 (21.6) & $34(3.7)$ & $230(24.9)$ \\
\hline Female & 1271 & $272(21.4)$ & $591(46.5)$ & 431 (33.9) & $417(32.8)$ & $310(24.4)$ & $64(5.0)$ & $359(28.2)$ \\
\hline$p$ value & & $<0.001$ & $<0.001$ & $<0.001$ & $<0.001$ & 0.133 & 0.129 & 0.080 \\
\hline
\end{tabular}

${ }^{\text {a }}$ Score for each domain ranges from 0 to 3 (no difficulty to severe difficulty), and a domain score $\geq 2$ indicates abnormal sleep in the domain

Table 3 Odds ratio (95\% confidence interval) of poor sleep quality associated with demographic, behavioral, and clinical factors

\begin{tabular}{|c|c|c|c|c|c|c|}
\hline \multirow[t]{2}{*}{ Factors } & \multicolumn{2}{|l|}{ Model $1^{\mathrm{a}}$} & \multicolumn{2}{|l|}{ Model $2^{\mathrm{a}}$} & \multicolumn{2}{|l|}{ Model $3^{\mathrm{a}}$} \\
\hline & Odds ratio $(95 \% \mathrm{CI})$ & $p$ & Odds ratio $(95 \% \mathrm{CI})$ & $p$ & Odds ratio $(95 \% \mathrm{CI})$ & $p$ \\
\hline Sex (female vs. male) & $1.72(1.39-2.13)$ & $<0.001$ & $2.09(1.47-2.96)$ & $<0.001$ & $1.76(1.46-2.12)$ & $<0.001$ \\
\hline Age (years) & $1.07(0.98-1.17)$ & 0.090 & $1.09(0.99-1.19)$ & 0.064 & - & - \\
\hline Education (years) & $0.94(0.82-1.09)$ & 0.471 & $0.92(0.79-1.07)$ & 0.287 & - & - \\
\hline Occupation (farmers vs. non-farmers) & $1.05(0.75-1.47)$ & 0.757 & $1.02(0.72-1.44)$ & 0.897 & - & - \\
\hline Marital status (married vs. others) & $1.00(0.81-1.24)$ & 0.937 & $0.97(0.78-1.21)$ & 0.848 & - & - \\
\hline Current smoking & $1.50(1.08-2.08)$ & 0.014 & $1.35(0.94-1.93)$ & 0.096 & - & - \\
\hline Current alcohol drinking & $1.11(0.85-1.44)$ & 0.445 & $0.97(0.72-1.31)$ & 0.887 & - & - \\
\hline Hypertension & $1.39(1.16-1.66)$ & $<0.001$ & $1.23(1.01-1.49)$ & 0.035 & $1.28(1.06-1.54)$ & 0.008 \\
\hline Hyperlipidemia & $1.32(1.04-1.68)$ & 0.020 & $1.03(0.80-1.34)$ & 0.789 & - & - \\
\hline Diabetes & $1.31(0.96-1.79)$ & 0.086 & $1.14(0.82-1.58)$ & 0.416 & - & - \\
\hline Coronary heart disease & $1.81(1.45-2.25)$ & $<0.001$ & $1.58(1.25-1.99)$ & $<0.001$ & $1.60(1.27-2.00)$ & $<0.001$ \\
\hline COPD & $1.99(1.47-2.71)$ & $<0.001$ & $1.78(1.30-2.44)$ & $<0.001$ & $1.82(1.34-2.49)$ & $<0.001$ \\
\hline Stroke & $1.44(1.08-1.92)$ & 0.011 & $1.29(0.96-1.74)$ & 0.090 & - & - \\
\hline
\end{tabular}

COPD chronic obstructive pulmonary disease

${ }^{a}$ Odds ratios (95\% confidence intervals, CIs) in Model 1 were derived from the logistic regression model that was adjusted for age, sex, and education, odds ratios (95\% CIs) in Model 2 were derived from the logistic regression model that included all these factors in the table, and odds ratios (95\% CIs) in Model 3 were derived from the best-fit model using the forward stepwise approaches

detected a marginally statistical interaction between gender and smoking on sleep disorders $(p$ for interaction $=0.10$ ). Stratifying analysis by gender showed that the age- and education-adjusted odds ratio of sleep disorders was $1.61(95 \%$ CI 1.08-2.41) in men and $1.30(0.73-2.31)$ in women, and the multiple-adjusted odds ratio was $1.40(0.90-2.17)$ in men and $1.18(0.61-2.26)$ in women. Finally, when using the forward stepwise multivariate analysis to fit the best model, the following factors showed significant associations with poor sleep quality: female sex $(\mathrm{OR}=1.76,95 \%$ CI 1.46-2.12), hypertension $(\mathrm{OR}=1.28,95 \%$ CI $1.06-1.54)$, CHD $(\mathrm{OR}=1.60,95 \%$ CI $1.27-2.00)$, and $\mathrm{COPD}(\mathrm{OR}=1.82,95 \%$ CI 1.34-2.49) (Table 3, model 3).

\section{Discussion}

In this large-scale population-based study, we showed that poor sleep quality affected nearly one-third of older adults who were living in the rural communities in China, which 
was slightly higher than that of those living in the urban areas $[5,6]$. The prevalence rates of sleep disturbance in the present study were $26.3 \%$ in men and $39.2 \%$ in women. These results are in contrast to some of the previous studies from urban areas, where elderly men had a higher prevalence of sleep disturbance than that of elderly women in China [5, 6]. Of note, more than $80 \%$ of our study population had no formal education or achieved primary school culture, and the majority of them were farmers, and about one-third had smoking and alcohol drinking hobbies, which might partly contribute to the higher prevalence rate in rural older adults, because research has indicated that rural people may ignore the importance of a healthy lifestyle and high quality sleep [29]. Among the seven PSQI domains, sleep latency was most highly prevalent (39.7\%), followed by sleep duration (31.0\%) and habitual sleep efficiency (28.8\%). These results were slightly different from those of other rural adults or the elderly in other provinces in China [29, 31]. Variations in diagnostic criteria, interview techniques, and age of the study populations may also partly contribute to the inconsistent results across studies.

In the present study, we found that female gender was associated with an increased likelihood of poor sleep quality, which is in line with the current view that women are more prone than men to experiencing sleep disorders [32-34]. The epidemiologic studies have indeed frequently indicated that female gender is an independent risk factor for sleep disorders $[35,36]$. The reason for poor sleep in women may be due to the fact that compared to men, women had higher proportions of low education, low individual income, and more chronic diseases, and were more susceptible to depression and anxiety [5, 31, 37].

In this study, suffering from some chronic diseases was associated with poor sleep quality, even after controlling for multiple potential confounders, such as hypertension, CHD, and COPD. A meta-analysis of 17 prospective cohort studies showed that short sleep duration, sleep continuity disturbance (SCD), early-morning awakening (EMA) and combined symptoms of insomnia were associated with an increased risk of hypertension [38]. Sleep-disordered breathing has been associated with chronically elevated blood pressure and hypertension in the US Wisconsin Sleep Cohort Study [39]. A systematic review and meta-analysis of prospective cohort studies indicated that compared with a sleep duration of $7 \mathrm{~h}$ per day, the risk of CHD increases by $11 \%$ per 1 -h decrease in sleep duration and increases by $7 \%$ per 1-h increase in sleep duration [40]. Moreover, a cohort study showed that participants with untreated severe sleep-disordered breathing (AHI > 30) were 2.6 times more likely to develop CHD or heart failure compared to those without sleep-disordered breathing [41]. A previous study showed that patients with higher COPD assessment test (CAT) scores generally had more severe insomnia [42].
Nighttime symptoms are usually common among patients with COPD, including trouble falling asleep, experienced nighttime awakenings, trouble staying asleep, and woke after a usual amount of sleep feeling worn out [43].

Several potential mechanisms might explain the potential link of sleep disorders with chronic diseases. First, the common mechanism connecting sleep disorders or poor sleep quality to the development of hypertension is that alterations in sleep quality/quantity lead to the loss of the nocturnal dip in blood pressure (BP) that is associated with non-rapid eye movement (NREM) sleep as the first step toward the development of hypertension. Further, loss of the BP dipping pattern is mainly attributable to an increase in nocturnal sympathetic activity, which in turn leads to a diurnally permanent increase in sympathetic tone, eventually mediates the occurrence of hypertension [44]. Second, abnormal sleep may lead to weight gain, metabolic disorders, inflammation, sympathetic activation, high blood clotting and endothelial dependent vasodilator dysfunction [40, 45]. Sleep-disordered breathing may cause oxidative stress, sympathetic activation, inflammation, hypercoagulability, endothelial dysfunction, and metabolic deregulation [46]. These can be linked with CHD. Third, patients with COPD are often accompanied by inadequate pulmonary ventilation and hypoventilation during sleep, then will lead to hypoxemia and hypercapnia, which have been associated with increased frequency of arousals during sleep [47]. Moreover, cough and wheeze [48], inhaled corticosteroid, and phlegm production are also associated with poor sleep quality [49].

Our population-based study targeted older residents living in less developed rural areas in China where little attention has been paid thus far by the research communities. Further, we used the internationally recognized and also validated scale in Chinese population to assess sleep quality and patterns. However, our study also has limitations. First, the cross-sectional nature of our study limited the possibility to make the causal inference. Second, lifestyle factors and medical disorders were assessed based on self-reported data, which might be subject to information bias. We ascertained the data from detailed questionnaire of health history including lifestyle factors, the time of first diagnosis and medication use of chronic diseases. Third, despite the adjustment for multiple potential confounding factors, other unknown or unmeasured confounding variables, such as physical activity [50], coffee or tea consumption, anxiety, and depression were not available [51].

In summary, poor sleep quality or sleep disorders are highly prevalent in a rural elderly population in China. In addition, some manageable behavioral factors and chronic health conditions were associated with a poor sleep quality. Potential influencing factors and the comprehensive intervention for sleep disorders are important topics for future research. 
Acknowledgements The authors would like to thank all the study participants for their valuable contribution to this project. Dr. C Qiu from Karolinska Institutet in Stockholm, Sweden provided valuable comments and editorial revisions on the manuscript.

Author contributions PW, LS, YW, ST, and YD designed and initiated the study. YW, XH, and YD assisted in data collection. PW and $\mathrm{XH}$ performed the statistical analysis. PW wrote the first draft of the article. All authors made critical comments on the manuscript and took part in interpretation of the results. YD, ST, and ZY supervised the study.

Funding The study was supported in part by research grants from the National Key R\&D Program of China (No.: 2017YFC1310100), the National Natural Science Foundation of China (No.: 81772448, 81501099, and 81861138008), and the Key R\&D Program of Shandong Province (No.: 2016ZDJS07A11), China.

\section{Compliance with ethical standards}

Conflict of interest The authors have no conflicts of interest to declare.

Ethical approval This study was approved by the Ethics Committee of Shandong Provincial Hospital Affiliated to Shandong University, Jinan, Shandong. Research has been conducted in accordance with the declaration of Helsinki and its later amendments.

Statement of human and animal rights This article does not contain any studies with animals performed by any of the authors.

Informed consent Written informed consent was obtained from all the participants of this study.

Open Access This article is distributed under the terms of the Creative Commons Attribution 4.0 International License (http://creativeco mmons.org/licenses/by/4.0/), which permits unrestricted use, distribution, and reproduction in any medium, provided you give appropriate credit to the original author(s) and the source, provide a link to the Creative Commons license, and indicate if changes were made.

\section{References}

1. National Bureau of Statistics of China (2018) Statistical Communique of the People's Republic of China on the 2017 National Economic and Social Development. http://www.stats.gov.cn/engli sh../PressRelease/201802/t20180228_1585666.html. Accessed 15 Jan 2019

2. van de Straat V, Bracke P (2015) How well does Europe sleep? A cross-national study of sleep problems in European older adults. Int J Public Health 60:643-650

3. Endeshaw YW, Yoo W (2016) Association between social and physical activities and insomnia symptoms among communitydwelling older adults. J Aging Health 28:1073-1089

4. Akbaraly TN, Jaussent I, Besset A et al (2015) Sleep complaints and metabolic syndrome in an elderly population: the Three-City Study. Am J Geriatr Psychiatry 23:818-828

5. Wang YM, Chen HG, Song M et al (2016) Prevalence of insomnia and its risk factors in older individuals: a community-based study in four cities of Hebei Province, China. Sleep Med 19:116-122

6. Luo J, Zhu G, Zhao Q et al (2013) Prevalence and risk factors of poor sleep quality among Chinese elderly in an urban community: results from the Shanghai aging study. PLoS One 8:e81261
7. Takada S, Shimizu YY (2018) Association between subjective sleep quality and future risk of falls in older people: results from LOHAS. J Gerontol A Biol Sci Med Sci 73:1205-1211

8. Chien MY, Chen HC (2015) Poor sleep quality is independently associated with physical disability in older adults. J Clin Sleep Med 11:225-232

9. Santos-Silva R, Castro LS, Taddei JA et al (2012) Sleep disorders and demand for medical services: evidence from a populationbased longitudinal study. PLoS One 7:e30085

10. Cai H, Shu XO, Xiang YB et al (2015) Sleep duration and mortality: a prospective study of 113138 middle-aged and elderly Chinese men and women. Sleep 38:529-536

11. Stranges S, Tigbe W, Gomez-Olive FX et al (2012) Sleep problems: an emerging global epidemic? Findings from the INDEPTH WHO-SAGE study among more than 40,000 older adults from 8 countries across Africa and Asia. Sleep 35:1173-1181

12. Wang S, Wu Y, Ungvari GS et al (2017) Sleep duration and its association with demographics, lifestyle factors, poor mental health and chronic diseases in older Chinese adults. Psychiatry Res 257:212-218

13. Sagayadevan V, Abdin E, Binte Shafie S et al (2017) Prevalence and correlates of sleep problems among elderly Singaporeans. Psychogeriatrics 17:43-51

14. Park J, Han JW, Lee JR et al (2018) Lifetime coffee consumption, pineal gland volume, and sleep quality in late life. Sleep. https:// doi.org/10.1093/sleep/zsy127

15. Watson EJ, Coates AM, Kohler M et al (2016) Caffeine consumption and sleep quality in Australian adults. Nutrients. https://doi. org/10.3390/nu8080479

16. Beland SG, Preville M, Dubois MF et al (2011) The association between length of benzodiazepine use and sleep quality in older population. Int J Geriatr Psychiatry 26:908-915

17. Qureshi A, Lee-Chiong $\operatorname{T~Jr}$ (2004) Medications and their effects on sleep. Med Clin N Am 88:751-766, x

18. Foral P, Knezevich J, Dewan N et al (2011) Medication-induced sleep disturbances. Consult Pharm 26:414-425

19. Ebrahim IO, Shapiro CM, Williams AJ et al (2013) Alcohol and sleep I: effects on normal sleep. Alcohol Clin Exp Res 37:539-549

20. Pan CW, Cong X, Zhou HJ et al (2017) Self-reported sleep quality, duration, and health-related quality of life in older Chinese: evidence from a rural town in Suzhou, China. J Clin Sleep Med 13:967-974

21. Li YVA, Fernandez-Mendoza J (2015) Insomnia with physiological hyperarousal is associated with hypertension. Hypertension 65:644-650

22. Cappuccio FP, D'Elia L, Strazzullo P et al (2010) Quantity and quality of sleep and incidence of type 2 diabetes: a systematic review and meta-analysis. Diabetes Care 33:414-420

23. Cappuccio FP, Cooper D, D'Elia L et al (2011) Sleep duration predicts cardiovascular outcomes: a systematic review and metaanalysis of prospective studies. Eur Heart J 32:1484-1492

24. Phua CS, Jayaram L, Wijeratne T (2017) Relationship between sleep duration and risk factors for stroke. Front Neurol 8:392

25. Cheng W, Rolls ET, Ruan H et al (2018) Functional connectivities in the brain that mediate the association between depressive problems and sleep quality. JAMA Psychiatry 75:1052-1061

26. Sindi S, Kåreholt I, Johansson L et al (2018) Sleep disturbances and dementia risk: a multicenter study. Alzheimer's Dement 14:1235-1242

27. Carroll JE, Seeman TE, Olmstead R et al (2015) Improved sleep quality in older adults with insomnia reduces biomarkers of disease risk: pilot results from a randomized controlled comparative efficacy trial. Psychoneuroendocrinology 55:184-192

28. Buysse DJ, Reynolds CF 3rd, Monk TH et al (1989) The Pittsburgh Sleep Quality Index: a new instrument for psychiatric practice and research. Psychiatry Res 28:193-213 
29. Li J, Yao YS, Dong Q et al (2013) Characterization and factors associated with sleep quality among rural elderly in China. Arch Gerontol Geriatr 56:237-243

30. Tsai PS, Wang SY, Wang MY et al (2005) Psychometric evaluation of the Chinese version of the Pittsburgh Sleep Quality Index (CPSQI) in primary insomnia and control subjects. Qual Life Res 14:1943-1952

31. Dong X, Wang Y, Chen Y et al (2018) Poor sleep quality and influencing factors among rural adults in Deqing, China. Sleep Breath 22:1213-1220

32. Madrid-Valero JJ, Martinez-Selva JM, Ribeiro do Couto B et al (2017) Age and gender effects on the prevalence of poor sleep quality in the adult population. Gaceta sanitaria 31:18-22

33. Liu X, Liu L (2005) Sleep habits and insomnia in a sample of elderly persons in China. Sleep 28:1579-1587

34. Quan SA, Li YC, Li WJ et al (2016) Gender differences in sleep disturbance among elderly Koreans: Hallym Aging Study. J Korean Med Sci 31:1689-1695

35. Quan SFKR, Olson J (2005) Factors associated with incidence and persistence of symptoms of disturbed sleep in an elderly cohort: the Cardiovascular Health Study. Am J Med Sci 329:163-172

36. Fok MSR, Besset A (2010) Incidence and persistence of sleep complaints in a community older population. Int J Geriatr Psychiatry 25:37-45

37. Leblanc MF, Desjardins S, Desgagne A (2015) Sleep problems in anxious and depressive older adults. Psychol Res Behav Manag 8:161-169

38. Meng L, Zheng Y, Hui R (2013) The relationship of sleep duration and insomnia to risk of hypertension incidence: a meta-analysis of prospective cohort studies. Hypertens Res 36:985-995

39. Peppard PE, Young T, Palta M et al (2000) Prospective study of the association between sleep-disordered breathing and hypertension. N Engl J Med 342:1378-1384

40. Wang D, Li W, Cui X et al (2016) Sleep duration and risk of coronary heart disease: a systematic review and meta-analysis of prospective cohort studies. Int J Cardiol 219:231-239

41. Hla KM, Young T, Hagen EW et al (2015) Coronary heart disease incidence in sleep disordered breathing: the Wisconsin Sleep Cohort Study. Sleep 38:677-684
42. Ban WH, Joo H, Lim JU et al (2018) The relationship between sleep disturbance and health status in patients with COPD. Int $\mathbf{J}$ Chronic Obstr Pulm Dis 13:2049-2055

43. Ding B, Small M, Bergstrom G et al (2017) A cross-sectional survey of night-time symptoms and impact of sleep disturbance on symptoms and health status in patients with COPD. Int J Chron Obstruct Pulmon Dis 12:589-599

44. Pepin JL, Borel AL, Tamisier R et al (2014) Hypertension and sleep: overview of a tight relationship. Sleep Med Rev 18:509-519

45. Bain AR, Weil BR, Diehl KJ et al (2017) Insufficient sleep is associated with impaired nitric oxide-mediated endotheliumdependent vasodilation. Atherosclerosis 265:41-46

46. Sánchez-de-la-Torre M, Campos-Rodriguez F, Barbé F (2013) Obstructive sleep apnoea and cardiovascular disease. Lancet Respir Med 1:61-72

47. Cormick WOL, Hensley MJ (1986) Nocturnal hypoxaemia and quality of sleep in patients with chronic obstructive lung disease. Thorax 41:846-854

48. Klink MEDR, Quan SF (1994) The relation of sleep complaints to respiratory symptoms in a general population. Chest 105:151-154

49. Chang CH, Chuang LP, Lin SW et al (2016) Factors responsible for poor sleep quality in patients with chronic obstructive pulmonary disease. BMC Pulm Med 16:118

50. Tsunoda K, Kitano N, Kai Y et al (2015) Prospective study of physical activity and sleep in middle-aged and older adults. Am J Prev Med 48:662-673

51. Smagula SF, Stone KL, Fabio A et al (2016) Risk factors for sleep disturbances in older adults: Evidence from prospective studies. Sleep Med Rev 25:21-30

Publisher's Note Springer Nature remains neutral with regard to jurisdictional claims in published maps and institutional affiliations. 